IRA-International Journal of Applied Sciences ISSN 2455-4499; Vol.04, Issue 03 (2016)

Institute of Research Advances

Pg. no. 506-518

http://research-advances.org/index.php/IRAJAS

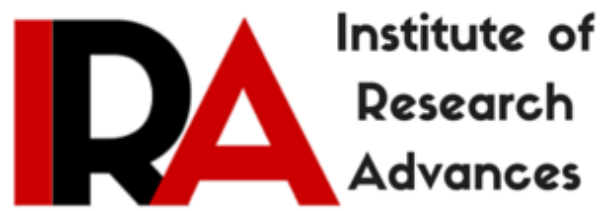

\title{
Comparative Limnological Studies in Mysuru City
}

\author{
Shiva Kumar $D^{1}$, Abhilash M R ${ }^{1}$, Nagaraju $A^{2}$, Smitha $N^{3}$ and Shivalingaiah ${ }^{2}$ \\ ${ }^{1}$ Department of Studies in Environmental Science, University of Mysore, \\ Manasagangothri, Mysuru-570006, India. \\ 2 Department of Post Graduate Botany, Maharani's Science College for Women, \\ Mysuru-570005, India. \\ ${ }^{3}$ Department of Studies in Botany, University of Mysore, Manasagangothri, \\ Mysuru-570006, India.
}

Type of Review: Peer Reviewed.

DOI: http://dx.doi.org/10.21013/jas.v4.n3.p14

\section{How to cite this paper:}

Kumar D, Shiva, M R, Abhilash, A, Nagaraju, N, Smitha, \& C, Shivalingaiah

(2016). Comparative Limnological Studies in Mysuru City. IRA-International Journal of Applied Sciences (ISSN 2455-4499), 4(3), 506-518.

doi:http://dx.doi.org/10.21013/jas.v4.n3.p14

(C) Institute of Research Advances

(cc) EY-NC

This work is licensed under a Creative Commons Attribution-Non Commercial 4.0 International License subject to proper citation to the publication source of the work.

Disclaimer: The scholarly papers as reviewed and published by the Institute of Research Advances (IRA) are the views and opinions of their respective authors and are not the views or opinions of the IRA. The IRA disclaims of any harm or loss caused due to the published content to any party. 


\begin{abstract}
Water security is emerging as an important and imperative issue for India. Many Indian cities are experiencing moderate to severe water shortages due to understood effects of industrialization and urbanization. These shortages would be further provoked by population stress and irrigation requirements that are major factors related to water insecurity. Around 1.2 billion people, or almost one-fifth of the world's population, live in areas of physical scarcity and 500 million people are approaching this situation. Another 1.6 billion people or almost one quarter of the world's population, are facing economic water shortage. Water use is growing at more than twice the rate of population increase in the last century. The water bodies, rivers, lakes, dams and estuaries are continuously subject to dynamic state of change with respect to the geological age and geochemical characteristics. The dynamic balance in the aquatic ecosystem is upset by human activities, resulting in pollution which is manifested dramatically as fish kill, offensive taste, odor, color, and unchecked aquatic weeds. Consequences of such human activities and discharge of sewage water makes the existence of the lakes more vulnerable. It is the prime necessary to take immediate remedial action to prevent all anthropogenic activities in the studied lakes or else the lakes will become biologically barren and will be lost forever. In the present study an attempt was made to know the physical, chemical, characteristic of lake water of selected lakes of Mysuru city.
\end{abstract}

Key words: Limnology, Mysuru, Rivers, Lakes, Biological Oxygen Demand (BOD), Chemical Oxygen Demand (COD)

\title{
I Introduction
}

As a crucial subsystem of urban environment, urban rivers and lakes offer many kinds of ecological services which benefit the city dwellers. However, with the growing pace of urbanization and rapid increase of economy. Water is one of the important natural resources of the planet earth and essential for the survival of all forms of life. Water is one of the greatest looming commodities of the $21^{\text {st }}$ century. It underpins all aspects of human society, from ecology to agriculture to industry and it has no known substitutes. An appreciable part of it gets lodged in snowcaps and natural lakes and is subsequently utilizable, in case of the former through snowmelt reaching a river system. A sizeable part is retained in surface soils and used up through evapo-transpiration for biomass generation. Another part seeps into the ground feeding natural aquifers. A major part appears in the river system downstream and is drained into seas, unless captured in man-made storages or artificially diverted from natural courses. Water is recycled continuously through transpiration and evaporation from land, river systems and oceans, besides precipitation through condensation, rain and snow. Water on the earth is in motion through the hydrological cycle. The utilization of water for most of the users i.e. humans, animals or plants involve movement of water. The conflict between the growing human population and the unchanging supply of freshwater has already started and may get worse by the years to come. Around 1.2 billion people, or almost one-fifth of the world's population, live in areas of physical scarcity and 500 million people are approaching this situation. Another 1.6 billion people or almost one quarter of the world's population, are facing economic water shortage (where countries lack the necessary infrastructure to take water from rivers and aquifers). Water use is growing at more than twice the rate of population increase in the last century, and, although there is no global water scarcity as such, an increasing number of regions are chronically short of water. Water quality characteristic of aquatic environment arise from a multitude of physical, chemical and biological interactions (Deuzane,.1979; Dee, 1989). The water bodies, rivers, lakes, dams and estuaries are continuously subject to dynamic state of change with respect to the geological age and geochemical characteristics. The dynamic balance in the aquatic ecosystem is upset by human activities, resulting in pollution which is manifested dramatically as fish kill, offensive taste, odor, color, and unchecked aquatic weeds. Lakes and reservoirs are important sources of surface water and livelihoods to many rural and urban communities. However, declining water quality in freshwater lakes and reservoirs is an increasing problem that threatens the ecosystem services to the riparian communities, especially in developing countries. One of the major causes of the decline in the quality of water is nutrient enrichment; mainly phosphorus and nitrogen. As a result, massive algal blooms may occur causing a 
shift from clear to a turbid state in shallow lakes and reservoirs (Kitaka et al., 2002; Lung'Ayia et al., 2000; Mugidde et al., 2005). Consequently, significant changes in the biological structure of the lakes and reservoirs occur which are a major threat to the sources of livelihoods to the riparian fisher folks (Harper, 1992). Nowadays, naturally existing dynamic equilibrium of water bodies like rivers, lakes and estuaries are affected by the human activities (Tamiru, 2004; Mahananda et al. 2010; Mehari and Mulu, 2013). In present scenario urban lakes are under direct threat of qualitative and quantitive degradation by means of pollution from both fixed-point and non-point sources. There are numerous sources of pollutants that could deteriorate the quality of water resources (Tamiru, 2004). Factors that are directly or indirectly polluting the lake ecosystems includes population growth, unplanned growth of city area, urbanization, agricultural land expansion and lack of awareness among the local residents. All such activities and pollution causing factors are decreasing the utility of water day by day (Tank and Chippa, 2013). Accessible man maid urban lakes and all other natural wetlands have gained the thrust of conservation in recent years due to their important functions in different hydrological processes. To take decisions and formulate policies concerning conservation, management and sustainable use of lakes and water resources, accurate spatial inventory is required (Finlayson and Davidson, 1999). Thus, sequential and continuous interpretation of physical, chemical and biological status of water quality and characterization of the pollution sources along with quantitative contribution of significant pollution causing parameters is important for demeanor pollution control management of urban lakes (Zhou et al., 2011). This will generate baseline data of water resources to evaluate the rate of change in water quality over a period of time.

\section{Materials and Methods}

\section{Study location}

Mysore is a city in the Indian state of Karnataka, and the administrative seat of Mysore District, one of the largest districts in Karnataka. Mysore was the former capital of the Kingdom of Mysore. Mysore is located at $770 \mathrm{~m}$ above sea level at $12.18^{\circ} \mathrm{N} 76.42^{\circ} \mathrm{E}$ and is $135 \mathrm{~km}$ from Bangalore, the state capital.

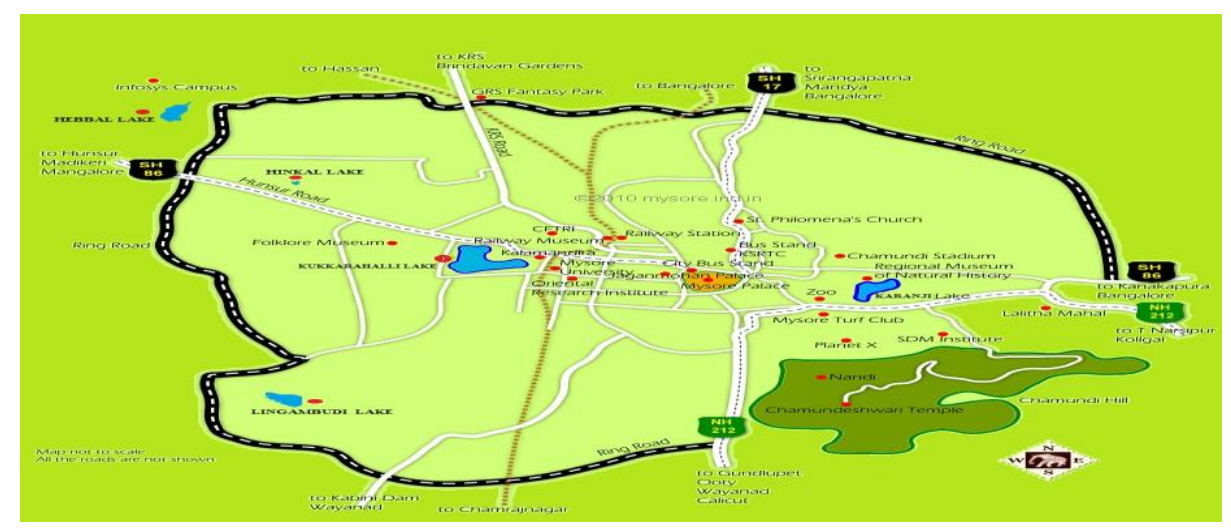

Figure 2: Location of selected lakes in Mysuru city

Station No 01: Kukkarali lake, Kukkaralli, Mysuru.

$12^{0} 18^{1} 27.9^{1} \mathrm{~N}$

$7^{0} 38^{1}{ }^{7.1} 1^{11} \mathrm{E}$

Station No 02: Karanji lake, Sidhartha layout, Mysuru.

$12^{0} 17^{1} 55.7^{1} \mathrm{~N}$ 


\title{
$76^{0} 40^{1} 9.8^{11} \mathrm{E}$
}

\section{Station No 03: Lingabuddi lake, Lingabudhi playa, Mysuru.}

\author{
$12^{0} 16^{1} 5.7^{1} \mathrm{~N}$
}

$76^{0} 36^{1} 46.1^{11} \mathrm{E}$

\section{Water Sampling}

It is often appropriate to match the sampling rate to the expected pattern of variation in the environment, to detect peak concentrations during short-term changes of water quality; eventcontrolled samplers are useful. The scope of environmental sampling addressed here will include the following aspects related to sampling: Where, When, What, How, and How Many to take samples; the choice of where (spatially) and when (temporally) to take samples generally should be based on sound statistics (simple random sampling, stratified random sampling, systematic sampling, and composite sampling) (Kratochvil et al., 1984). Although guidelines exist, there is no set rule regarding the number, the amount, and the frequency of samples/sampling. For instance, the optimum number of samples to collect is nearly always limited by the amount of resources available. The water samples were collected at different points in the lake for analysis, including at inflow and out flow points of the lake for different seasons, shows the location of lake. Clean and sterilized plastic cans of two litre capacity were used to collect the samples for analysis and also to determine dissolved oxygen, the Sterilized glass bottles were used. The analytical and average results of water samples are presented in table for both seasons. The results were compared with Indian drinking water standards and also the analysis was carried as per the procedure laid by standard method.

\section{Sample Preservation and Storage}

Three approaches (i.e., refrigeration, use of proper sample container, and addition of preserving chemicals) are generally used to minimize such changes, refrigeration (including freezing) is a universally applicable method to slow down all loss processes. The only exception that refrigeration does not help water samples are preserved for metal analysis, (Spellman, 2008). Cold storage will adversely reduce metal solubility and enhance precipitation in the solution. The proper selection of containers (material type and headspace) is critical to reduce losses through several physical processes, such as volatilization, adsorption, absorption, and diffusion. The addition of chemicals is essential to some parameters for their losses due to chemical reaction and bacterial degradation. Chemical addition or $\mathrm{pH}$ change can also be effective to reduce metal adsorption to glass container walls. Water samples from each location of the sampling stations were collected during four seasons (November, January, March and May) of 2015-2016 in five litres capacity polyethylene cans. All the samples were transported to laboratory and stored at $4^{\circ} \mathrm{C}$.

\section{Statistical Analysis:}

Correlation is a statistical measure of the interdependence of two or more random variables. Fundamentally, the value indicates how much of a change in one variable is explained by a change in another. The measurement scales used should be at least interval scales, but other correlation coefficients are available to handle other types of data. Correlation coefficients can range from -1 to +1 . The value of -1 . represents a perfect negative correlation and a value of +1 represents a perfect positive correlation. A value of 0.00 represents there is no relation between analyzed parameters. Statistical analysis of correlation coefficient was made using PAST software package. From the overall studies of statistical analysis, it can be concluded that, there is strong relationship between majorities of cations and anions along with physical parameters, which has a prominent role in the study of hydro chemical facies of lake water. 


\section{Result and Discussion}

Table 1: Physico-chemical and Biological characteristics of lake water

\begin{tabular}{|c|c|c|c|c|c|}
\hline \multirow{2}{*}{ Sl.No } & \multirow{2}{*}{ Parameters } & \multicolumn{4}{|c|}{ Kukkarahalli lake } \\
\hline & & November & January & March & May \\
\hline 1 & Colour & SG & SG & SG & SG \\
\hline 2 & Temperature & 27.5 & 25.5 & 26.4 & 26.1 \\
\hline 3 & pH & 9.3 & 8.9 & 8.7 & 9.0 \\
\hline 4 & EC & 1225 & 1110 & 640 & 610 \\
\hline 5 & Turbidity & 106.3 & 84.6 & 90.0 & 92.1 \\
\hline 6 & TDS & 785 & 682 & 426 & 395 \\
\hline 7 & TSS & 175.2 & 150.2 & 162.1 & 132.1 \\
\hline 8 & DO & 3.8 & 3.2 & 4.7 & 4.2 \\
\hline 9 & BOD & 12 & 15 & 9.2 & 9.9 \\
\hline 10 & COD & 49 & 32 & 68 & 55 \\
\hline 11 & Nitrate & 0.49 & 0.22 & 0.48 & 0.46 \\
\hline 12 & Total nitrogen & 2.96 & 3.48 & 3.84 & 2.86 \\
\hline 13 & Total phosphate & 5.21 & 5.02 & 5.82 & 6.71 \\
\hline 14 & Sulphate & 5.25 & 4.68 & 3.47 & 5.62 \\
\hline 15 & Calcium & 92.18 & 68.14 & 96.19 & 88.70 \\
\hline 16 & Magnesium & 220.36 & 300.18 & 118.3 & 129.7 \\
\hline 17 & Total hardness & 760.60 & 986.02 & 778.6 & 750.6 \\
\hline 18 & Sodium & 15.5 & 8.8 & 10.4 & 16.1 \\
\hline 19 & Potassium & 21.5 & 12.6 & 13.0 & 21.0 \\
\hline 20 & Chloride & 62.05 & 31.90 & 54.86 & 49.63 \\
\hline
\end{tabular}

Note: $\mathrm{SG}=$ slightly green, $\mathrm{PB}=$ Pinch block, $\mathrm{SB}=$ slightly brown

Table 2: Physico-chemical and Biological characteristics of lake water

\begin{tabular}{|c|l|c|c|c|c|}
\hline \multirow{2}{*}{ SI.No. } & \multirow{2}{*}{ Parameters } & \multicolumn{4}{|c|}{ Karanji lake } \\
\cline { 3 - 6 } & & November & January & March & May \\
\hline $\mathbf{1}$ & Colour & PB & PB & PB & PB \\
\hline $\mathbf{2}$ & Temperature & 27.3 & 26.3 & 28.0 & 26.5 \\
\hline $\mathbf{3}$ & pH & 9.1 & 8.7 & 7.7 & 9.2 \\
\hline $\mathbf{4}$ & EC & 804 & 840 & 520 & 480 \\
\hline $\mathbf{5}$ & Turbidity & 95.6 & 67.4 & 86.1 & 87.3 \\
\hline $\mathbf{6}$ & TDS & 515 & 438 & 270 & 308 \\
\hline $\mathbf{7}$ & TSS & 92.5 & 87.4 & 92.4 & 90.6 \\
\hline $\mathbf{8}$ & DO & 8.2 & 8.2 & 8.4 & 8.1 \\
\hline $\mathbf{9}$ & BOD & 6.1 & 2.3 & 2.4 & 2.0 \\
\hline $\mathbf{1 0}$ & COD & 36 & 45 & 45 & 35 \\
\hline
\end{tabular}




\begin{tabular}{|c|l|c|c|c|c|}
\hline $\mathbf{1 1}$ & Nitrate & 0.3 & 0.2 & 0.2 & 0.3 \\
\hline $\mathbf{1 2}$ & Total nitrogen & 2.95 & 1.93 & 1.86 & 2.8 \\
\hline $\mathbf{1 3}$ & Total phosphate & 6.55 & 3.58 & 4.23 & 5.23 \\
\hline $\mathbf{1 4}$ & Sulphate & 4.85 & 5.18 & 3.18 & 4.66 \\
\hline $\mathbf{1 5}$ & Calcium & 224.44 & 186.2 & 216.4 & 216.4 \\
\hline $\mathbf{1 6}$ & Magnesium & 66.07 & 53.07 & 76.3 & 66.0 \\
\hline $\mathbf{1 7}$ & Total hardness & 830.66 & 608.2 & 836.1 & 810.6 \\
\hline $\mathbf{1 8}$ & Sodium & 7.0 & 3.3 & 4.6 & 5.6 \\
\hline $\mathbf{1 9}$ & Potassium & 11.2 & 7.6 & 8.3 & 10.2 \\
\hline $\mathbf{2 0}$ & Chloride & 51.40 & 60.26 & 138.25 & 44.31 \\
\hline
\end{tabular}

Note: $\mathrm{SG}=$ slightly green, $\mathrm{PB}=$ Pinch block, $\mathrm{SB}=$ slightly brown

Table 3: Physico-chemical and Biological characteristics of lake water

\begin{tabular}{|c|c|c|c|c|c|}
\hline \multirow{2}{*}{ Sl.No. } & \multirow{2}{*}{ Parameters } & \multicolumn{4}{|c|}{ Lingambudi lake } \\
\hline & & November & January & March & May \\
\hline 1 & Colour & SB & SB & SB & SB \\
\hline 2 & Temperature & 24.0 & 27.4 & 26.4 & 22.1 \\
\hline 3 & pH & 7.8 & 7.8 & 8.2 & 8.4 \\
\hline 4 & EC & 956 & 990 & 820 & 585 \\
\hline 5 & Turbidity & 61.2 & 52.1 & 49.6 & 53.1 \\
\hline 6 & TDS & 612 & 562 & 365 & 375 \\
\hline 7 & TSS & 51.5 & 55.4 & 47.3 & 50.6 \\
\hline 8 & DO & 8.4 & 8.0 & 7.4 & 8.5 \\
\hline 9 & BOD & 3.1 & 4.2 & 2.8 & 3.4 \\
\hline 10 & COD & 54 & 38 & 51 & 53 \\
\hline 11 & Nitrate & 0.60 & 0.46 & 0.7 & 0.66 \\
\hline 12 & Total nitrogen & 2.96 & 1.96 & 2.8 & 2.86 \\
\hline 13 & Total phosphate & 5.75 & 4.44 & 4.51 & 5.55 \\
\hline 14 & Sulphate & 4.65 & 3.27 & 2.87 & 2.66 \\
\hline 15 & Calcium & 300.6 & 250.6 & 218.1 & 292.5 \\
\hline 16 & Magnesium & 95.71 & 85.4 & 167 & 56.3 \\
\hline 17 & Total hardness & 980.78 & 881.46 & 583.2 & 960.7 \\
\hline 18 & Sodium & 8.8 & 5.8 & 8.6 & 6.1 \\
\hline 19 & Potassium & 12.8 & 10.7 & 13.2 & 11.0 \\
\hline 20 & Chloride & 49.63 & 134.71 & 38.99 & 46.08 \\
\hline
\end{tabular}

Note: $\mathrm{SG}=$ slightly green, $\mathrm{PB}=$ Pinch block, $\mathrm{SB}=$ slightly brown 
IRA-International Journal of Applied Sciences

Table 4: Pearson correlation matrix for the analysed parameters of Kukkarahalli lake.

\begin{tabular}{|c|c|c|c|c|c|c|c|c|c|c|c|c|c|c|c|c|c|c|c|}
\hline & Тетp & $p H$ & $E C$ & $\begin{array}{c}\text { Turbidit } \\
y\end{array}$ & $T D S$ & $T S S$ & $D O$ & $B O D$ & $C O D$ & $\begin{array}{c}\text { Nitrat } \\
e\end{array}$ & $\begin{array}{c}\text { nitroge } \\
n\end{array}$ & $\begin{array}{c}\text { Phosphat } \\
e \\
\end{array}$ & $\begin{array}{c}\text { Sulphat } \\
e\end{array}$ & $\mathrm{Ca}$ & $M g$ & $\begin{array}{c}\text { hardnes } \\
s\end{array}$ & $\mathrm{Na}$ & $K$ & $\begin{array}{l}C \\
l \\
\end{array}$ \\
\hline Temp & 1 & & & & & & & & & & & & & & & & & & \\
\hline $\mathrm{pH}$ & 0.664 & 1 & & & & & & & & & & & & & & & & & \\
\hline $\mathrm{EC}$ & 0.320 & 0.648 & 1 & & & & & & & & & & & & & & & & \\
\hline Turbidity & 0.966 & 0.829 & 0.409 & 1 & & & & & & & & & & & & & & & \\
\hline TDS & 0.391 & 0.672 & 0.996 & 0.471 & 1 & & & & & & & & & & & & & & \\
\hline TSS & 0.725 & 0.320 & 0.594 & 0.606 & 0.645 & 1 & & & & & & & & & & & & & \\
\hline DO & 0.274 & $\begin{array}{c}- \\
0.404 \\
\end{array}$ & $\begin{array}{c}- \\
0.784 \\
\end{array}$ & 0.102 & $\begin{array}{c}- \\
0.735 \\
\end{array}$ & 0.005 & 1 & & & & & & & & & & & & \\
\hline BOD & $\begin{array}{c}- \\
0.323 \\
\end{array}$ & 0.252 & 0.791 & -0.199 & 0.741 & 0.095 & $\begin{array}{c}- \\
0.973 \\
\end{array}$ & 1 & & & & & & & & & & & \\
\hline COD & 0.364 & -0.33 & $\begin{array}{c}- \\
0.719 \\
\end{array}$ & 0.188 & $\begin{array}{c}- \\
0.664 \\
\end{array}$ & 0.097 & 0.994 & -0.96 & 1 & & & & & & & & & & \\
\hline Nitrate & 0.753 & 0.230 & $\begin{array}{c}- \\
0.379 \\
\end{array}$ & 0.671 & $\begin{array}{c}- \\
0.309\end{array}$ & 0.265 & 0.795 & -0.85 & 0.837 & 1 & & & & & & & & & \\
\hline nitrogen & -0.35 & -0.82 & $\begin{array}{c}- \\
0.196 \\
\end{array}$ & -0.568 & $\begin{array}{c}- \\
0.203 \\
\end{array}$ & 0.244 & 0.243 & $0 . \overline{-} 1$ & 0.227 & -0.255 & 1 & & & & & & & & \\
\hline phosphate & $0 . \overline{120}$ & $\begin{array}{c}- \\
0.203 \\
\end{array}$ & $\begin{array}{c}- \\
0.865 \\
\end{array}$ & -0.09 & -0.85 & -0.69 & 0.640 & $\begin{array}{c}- \\
0.759 \\
\end{array}$ & 0.585 & 0.506 & -0.320 & 1 & & & & & & & \\
\hline Sulphate & 0.149 & 0.768 & 0.255 & 0.394 & 0.243 & $\begin{array}{c}- \\
0.359 \\
\end{array}$ & $\overline{-}-\overline{427}$ & 0.211 & -0.42 & 0.019 & -0.970 & 0.246 & 1 & & & & & & \\
\hline Calcium & 0.703 & 0.065 & -0.43 & 0.573 & $\begin{array}{c}- \\
0.364 \\
\end{array}$ & 0.322 & 0.873 & $\begin{array}{c}- \\
0.893 \\
\end{array}$ & 0.912 & 0.979 & -0.057 & 0.458 & -0.180 & 1 & & & & & \\
\hline $\begin{array}{c}\text { Magnesiu } \\
\text { m }\end{array}$ & -0.22 & 0.309 & 0.852 & -0.107 & 0.810 & 0.204 & $\begin{array}{c}- \\
0.962 \\
\end{array}$ & 0.993 & $0 . \overline{9} 5$ & -0.805 & -0.021 & -0.817 & 0.194 & $\begin{array}{c}- \\
0.837\end{array}$ & 1 & & & & \\
\hline hardness & $\begin{array}{c}- \\
0.689 \\
\end{array}$ & $\begin{array}{c}- \\
0.263 \\
\end{array}$ & 0.436 & -0.642 & 0.371 & -0.11 & $\begin{array}{c}- \\
0.769 \\
\end{array}$ & 0.866 & $\begin{array}{c}- \\
0.800 \\
\end{array}$ & -0.984 & 0.377 & -0.621 & -0.15 & $\begin{array}{c}- \\
0.939 \\
\end{array}$ & 0.825 & 1 & & & \\
\hline Sodium & 0.607 & 0.701 & -0.08 & 0.729 & $\begin{array}{c}- \\
0.047 \\
\end{array}$ & $\begin{array}{c}- \\
0.094 \\
\end{array}$ & 0.234 & -0.43 & 0.262 & 0.690 & -0.874 & 0.533 & 0.736 & 0.532 & $\begin{array}{c}- \\
0.412 \\
\end{array}$ & -0.779 & 1 & & \\
\hline Potassium & 0.627 & 0.816 & 0.090 & 0.775 & 0.124 & $\begin{array}{c}- \\
0.029\end{array}$ & 0.066 & -0.27 & 0.103 & 0.59 & -0.927 & 0.389 & 0.809 & 0.417 & $\begin{array}{c}- \\
0.242\end{array}$ & -0.676 & $\begin{array}{c}0.98 \\
3\end{array}$ & 1 & \\
\hline Chloride & 0.916 & 0.407 & -0.08 & 0.835 & $\begin{array}{c}- \\
0.009\end{array}$ & 0.528 & 0.627 & $\begin{array}{c}- \\
0.675\end{array}$ & 0.695 & 0.950 & -0.259 & 0.224 & 0.020 & 0.927 & $\begin{array}{c}- \\
0.593\end{array}$ & -0.902 & $\begin{array}{c}0.65 \\
3\end{array}$ & $\begin{array}{c}0.59 \\
9\end{array}$ & 1 \\
\hline
\end{tabular}


IRA-International Journal of Applied Sciences

Table 5: Pearson correlation matrix for the analysed parameters of Karanji lake.

\begin{tabular}{|c|c|c|c|c|c|c|c|c|c|c|c|c|c|c|c|c|c|c|c|}
\hline & Тетp & $p H$ & $E C$ & $\begin{array}{c}\text { Turbidit } \\
y\end{array}$ & $T D S$ & TSS & $D O$ & $B O D$ & $C O D$ & $\begin{array}{c}\text { Nitrat } \\
e\end{array}$ & $\begin{array}{c}\text { nitroge } \\
n\end{array}$ & $\begin{array}{c}\text { Phosphat } \\
e \\
\end{array}$ & $\begin{array}{c}\text { Sulphat } \\
e\end{array}$ & $\mathrm{Ca}$ & $M g$ & $\begin{array}{c}\text { hardnes } \\
\quad s\end{array}$ & $\mathrm{Na}$ & K & $\begin{array}{l}C \\
l\end{array}$ \\
\hline Temp & 1 & & & & & & & & & & & & & & & & & & \\
\hline $\mathrm{pH}$ & $\begin{array}{c}- \\
0.703 \\
\end{array}$ & 1 & & & & & & & & & & & & & & & & & \\
\hline EC & $\begin{array}{c}- \\
0.303 \\
\end{array}$ & 0.279 & 1 & & & & & & & & & & & & & & & & \\
\hline Turbidity & 0.557 & 0.171 & $\begin{array}{c}- \\
0.329 \\
\end{array}$ & 1 & & & & & & & & & & & & & & & \\
\hline TDS & $0 . \overline{2} 79$ & 0.548 & 0.911 & 0.032 & 1 & & & & & & & & & & & & & & \\
\hline TSS & 0.824 & $\begin{array}{c}- \\
0.209 \\
\end{array}$ & -0.41 & 0.926 & -0.15 & 1 & & & & & & & & & & & & & \\
\hline DO & 0.840 & $\begin{array}{c}- \\
0.957 \\
\end{array}$ & -0.14 & 0.017 & -0.35 & 0.386 & 1 & & & & & & & & & & & & \\
\hline BOD & 0.286 & 0.341 & 0.535 & 0.619 & 0.775 & 0.501 & $0 . \overline{-}$ & 1 & & & & & & & & & & & \\
\hline COD & 0.215 & -0.80 & 0.169 & -0.68 & -0.23 & -0.37 & 0.710 & $\begin{array}{c}- \\
0.440 \\
\end{array}$ & 1 & & & & & & & & & & \\
\hline Nitrate & $\begin{array}{c}- \\
0.184 \\
\end{array}$ & 0.800 & -0.11 & 0.712 & 0.291 & 0.399 & -0.68 & 0.505 & $\begin{array}{c}- \\
0.997 \\
\end{array}$ & 1 & & & & & & & & & \\
\hline nitrogen & $\begin{array}{c}- \\
0.183 \\
\end{array}$ & 0.818 & $\begin{array}{c}- \\
0.005 \\
\end{array}$ & 0.706 & 0.399 & 0.389 & -0.68 & 0.593 & $\begin{array}{c}- \\
0.982 \\
\end{array}$ & 0.992 & 1 & & & & & & & & \\
\hline phosphate & 0.192 & 0.562 & 0.047 & 0.881 & 0.444 & 0.665 & -0.34 & 0.811 & -0.85 & 0.885 & 0.913 & 1 & & & & & & & \\
\hline Sulphate & $\begin{array}{c}- \\
0.853 \\
\end{array}$ & 0.845 & 0.661 & -0.29 & 0.730 & -0.61 & -0.82 & 0.245 & -0.36 & 0.375 & 0.428 & 0.1795 & 1 & & & & & & \\
\hline Calcium & 0.610 & 0.076 & -0.44 & 0.990 & -0.10 & 0.951 & 0.086 & 0.514 & -0.63 & 0.654 & 0.633 & 0.8104 & -0.41 & 1 & & & & & \\
\hline $\begin{array}{l}\text { Magnesiu } \\
\text { m }\end{array}$ & 0.872 & -0.52 & -0.70 & 0.698 & -0.57 & 0.887 & 0.591 & 0.064 & $\begin{array}{c}- \\
0.081\end{array}$ & 0.081 & 0.031 & 0.2781 & -0.888 & $\begin{array}{c}0.78 \\
2\end{array}$ & 1 & & & & \\
\hline hardness & 0.691 & -0.09 & $\begin{array}{c}- \\
0.601\end{array}$ & 0.937 & $\begin{array}{c}- \\
0.305\end{array}$ & 0.961 & 0.218 & 0.345 & $\begin{array}{c}- \\
0.512\end{array}$ & 0.519 & 0.481 & 0.666 & -0.583 & $\begin{array}{c}0.97 \\
6\end{array}$ & $\begin{array}{c}0.89 \\
0\end{array}$ & 1 & & & \\
\hline Sodium & 0.294 & 0.470 & -0.08 & 0.939 & 0.319 & 0.756 & $\begin{array}{c}- \\
0.258 \\
\end{array}$ & 0.760 & $\begin{array}{c}- \\
0.837 \\
\end{array}$ & 0.866 & 0.883 & 0.989 & 0.044 & $\begin{array}{c}0.88 \\
6 \\
\end{array}$ & $\begin{array}{c}0.41 \\
0 \\
\end{array}$ & 0.766 & 1 & & \\
\hline Potassium & 0.078 & 0.647 & -0.05 & 0.859 & 0.357 & 0.60 & $\begin{array}{c}- \\
0.465 \\
\end{array}$ & 0.697 & $0 . \overline{933}$ & 0.954 & 0.965 & 0.982 & 0.221 & $\begin{array}{c}0.79 \\
7 \\
\end{array}$ & $\begin{array}{c}0.25 \\
0 \\
\end{array}$ & 0.660 & $\begin{array}{c}0.97 \\
5 \\
\end{array}$ & 1 & \\
\hline Chloride & 0.802 & $\begin{array}{c}- \\
0.983 \\
\end{array}$ & $\begin{array}{c}- \\
0.382\end{array}$ & 0.001 & $\begin{array}{c}- \\
0.589\end{array}$ & $\begin{array}{c}0.374 \\
9\end{array}$ & 0.963 & -0.27 & 0.683 & -0.68 & -0.704 & -0.425 & -0.92 & $\begin{array}{c}0.10 \\
1 \\
\end{array}$ & $\begin{array}{c}0.67 \\
1 \\
\end{array}$ & 0.272 & -0.31 & $\begin{array}{c}- \\
0.507\end{array}$ & 1 \\
\hline
\end{tabular}


IRA-International Journal of Applied Sciences

Table 6: Pearson correlation matrix for the analysed parameters of Lingambudi lake.

\begin{tabular}{|c|c|c|c|c|c|c|c|c|c|c|c|c|c|c|c|c|c|c|c|}
\hline & Temp & $p H$ & $E C$ & Turbidity & $T D S$ & TSS & $D O$ & $B O D$ & $C O D$ & Nitrate & nitrogen & Phosphate & Sulphate & $\mathrm{Ca}$ & $M g$ & hardness & $\mathrm{Na}$ & $K$ & $\mathrm{Cl}$ \\
\hline Temp & 1 & & & & & & & & & & & & & & & & & & \\
\hline $\mathrm{pH}$ & -0.531 & 1 & & & & & & & & & & & & & & & & & \\
\hline EC & 0.725 & -0.959 & 1 & & & & & & & & & & & & & & & & \\
\hline Turbidity & -0.425 & -0.509 & 0.313 & 1 & & & & & & & & & & & & & & & \\
\hline TDS & 0.228 & -0.940 & 0.809 & 0.729 & 1 & & & & & & & & & & & & & & \\
\hline TSS & 0.253 & -0.640 & 0.487 & 0.236 & 0.705 & 1 & & & & & & & & & & & & & \\
\hline DO & -0.747 & -0.033 & -0.248 & 0.674 & 0.366 & 0.432 & 1 & & & & & & & & & & & & \\
\hline BOD & 0.319 & -0.396 & 0.292 & -0.114 & 0.413 & 0.932 & 0.274 & 1 & & & & & & & & & & & \\
\hline COD & -0.753 & 0.477 & -0.519 & 0.397 & -0.314 & -0.738 & 0.251 & -0.855 & 1 & & & & & & & & & & \\
\hline Nitrate & -0.490 & 0.751 & -0.660 & -0.144 & -0.730 & -0.965 & -0.200 & -0.903 & 0.844 & 1 & & & & & & & & & \\
\hline nitrogen & -0.715 & 0.459 & -0.487 & 0.387 & -0.312 & -0.762 & 0.203 & -0.882 & 0.998 & 0.854 & 1 & & & & & & & & \\
\hline phosphate & -0.891 & 0.116 & -0.336 & 0.788 & 0.199 & -0.079 & 0.830 & -0.301 & 0.729 & 0.283 & 0.699 & 1 & & & & & & & \\
\hline Sulphate & -0.025 & -0.767 & 0.657 & 0.908 & 0.858 & 0.261 & 0.342 & -0.101 & 0.183 & -0.278 & 0.195 & 0.475 & 1 & & & & & & \\
\hline Calcium & -0.782 & -0.078 & -0.193 & 0.803 & 0.411 & 0.3036 & 0.973 & 0.086 & 0.411 & -0.08 & 0.370 & 0.922 & 0.493 & 1 & & & & & \\
\hline Magnesium & 0.563 & -0.012 & 0.274 & -0.365 & -0.272 & -0.633 & -0.910 & -0.594 & 0.093 & 0.411 & 0.148 & -0.541 & -0.051 & -0.791 & 1 & & & & \\
\hline hardness & -0.568 & -0.252 & -0.029 & 0.705 & 0.555 & 0.622 & 0.970 & 0.439 & 0.052 & -0.422 & 0.008 & 0.719 & 0.443 & 0.931 & -0.915 & 1 & & & \\
\hline Sodium & 0.017 & -0.156 & 0.260 & 0.375 & 0.085 & -0.640 & -0.329 & -0.842 & 0.612 & 0.532 & 0.653 & 0.202 & 0.530 & -0.103 & 0.689 & -0.375 & 1 & & \\
\hline Potassium & 0.072 & -0.039 & 0.184 & 0.203 & -0.071 & -0.739 & -0.465 & -0.883 & 0.594 & 0.614 & 0.639 & 0.080 & 0.373 & -0.251 & 0.789 & -0.528 & 0.983 & 1 & \\
\hline Chloride & 0.622 & -0.593 & 0.564 & -0.164 & 0.504 & 0.889 & -0.01 & 0.935 & -0.964 & -0.952 & -0.971 & -0.527 & -0.001 & -0.164 & -0.295 & 0.201 & -0.64 & -0.672 & 1 \\
\hline
\end{tabular}


Natural water body contains uniform water solution which undergoes unremitting physicochemical transformation due to circulation in the environment that greatly affects the water quality composition. Variation in results is found between different lakes as well as within different sites of the lakes. However there could be always a chance for difference in test result in different laboratories because of laboratory approach, sample preservation, quality of chemicals used and testing methods applied (Weldemarim, 2013). Results of the physicochemical parameters obtained from this study are discussed bellow. Turbidity is caused due to presence of suspended matter, clay silt, colloidal organic particles, plankton and other microscopic organisms. TDS is a measure of the solid materials dissolved in the water. This includes salts, some organic materials, and a wide range of other materials from nutrients to toxic materials.

The Total Suspended Solids indicates the amount of solid suspended in water. High concentration of particulate matter can cause increased sedimentation and siltation in a stream. Dissolved oxygen is essential for the self-purification process in natural water systems. The dissolved oxygen level may indicate the effects of oxidisable wastes on receiving waters. High amount of oxygen in the water body shows the good quality of water with penetration of sun light to the water. The Biological oxygen demand is an empirical test used to determine the relative oxygen requirement of water /wastewater. BOD provides an estimate of how much biodegradable waste is present in the water, whereas Chemical Oxygen Demand test is commonly used to indirect measure of the amount of organic compound in water/wastwater.

Nitrate is basically non- toxic but when ingested with food or water it will be reduced by bacterial action. Nitrates are produced during the final stage of decomposition of organic matter. Presence of nitrate in water indicates contamination by runoff containing fertilizer or human and animal wastes. Phosphorous is essential to the growth of organisms and can be nutrient that limits the primary productivity of a body of water (APHA, 2005). Sulphates in natural water system may find its origin both from natural such as leaching from rocks with deposition of gypsum, oxidation of organic materials from household origin as well as industrial sources. The permanent hardness is due to carbonates, sulphates and chlorides of calcium and magnesium. Sodium salts are highly soluble in water and hence all natural waters contain a little amount of sodium (Roberts and Marsh, 1987).

The quality of water is also depending on the concentration of potassium present in the sample Chloride ion is generally present in natural water and its presence can be attributed to the dissolution of salt deposits, discharges, irrigation drainage and contamination from refuge leachates. The salty taste produced but chloride ion depends on chemical composition of the water.

\section{Conclusion}

Water is one of the important natural resources of the planet earth and essential for the survival of all forms of life. An appreciable part of it gets lodged in snow caps and natural lakes and is subsequently utilizable, in case of the former through snowmelt reaching a river system. A sizeable part is retained in surface soils and used up through evapo-transpiration for biomass generation. Another part seeps into the ground feeding natural aquifers. Water is recycled continuously through transpiration and evaporation from land, river systems and oceans, besides precipitation through condensation, rain and snow. The study area of Mysuru City, whose growth rate is posed to be that of rapid, equipped with only primary treatment plants, treats only a part of UWW generated from the city limits. Large quantity of untreated UWW being discharged into low lying areas and water bodies located in and around the city. Due to the scarcity of water, the lake water is used for irrigation and domestic usage. In this research the findings shows the entry of the sewage and industrial effluent from different chemical processes. 


\section{References}

1. APHA, (2005). Standard methods for examination of water and wastewater; $21^{\text {st }}$ edition, New York.

2. Dee AK (1989). Environmental chemistry. Second ed. pp. 164-272.

3. Deuzuane J (1979). Handbook of drinking water quality. Indiana Univ. Press pp. 3-17.

4. Finlayson, C.M. and Davidson, N.C. (collators), 1999. Global Review of Wetland Resources and Priorities for Wetland Inventory: Summary report, in Global Review of Wetland Resources and Priorities for Wetland Inventory, Finlayson, C.M. and Spiers, A.G., Ed., CDROM, Supervising Scientist Report 144, Canberra, Australia.

5. G. Roberts and T. Marsh, "The effect of agricultural practices on the nitrate concentrations in the surface water domestic supply sources of western Europe," IAHS, vol. 164, pp. 365-380, 1987.

6. Harper, D. (1992). The ecological relationships of aquatic plants at Lake Naivasha, Kenya. Hydrobiologia 232, 65-71.

7. Kitaka, N., Harper, D. M., and Mavuti, K. M. (2002). Phosphorus inputs to Lake Naivasha, Kenya, from its catchment and the trophic state of the lake. Hydrobiologia 488, 73-80.

8. Kratochvil, B., Wallace, D. \& Taylor, J. K. 1984. Sampling for chemical analysis. Anal. Chem. 56: 113R-129R

9. Lung'Ayia, H., M'harzi, A., Tackx, M., Gichuki, J., and Symoens, J. (2000). Phytoplankton community structure and environment in the Kenyan waters of Lake Victoria. Freshwater Biology 43, 529-543.

10. Mahananda, M.R., Mohanty, B.P. and Behera, N.R., 2010 Physico-chemical analysis of surface and ground water of bargarh district, Orissa, india, International Journal of Research and Reviews in Applied Sciences, vol.2(3), pp. 284-295.

11. Mehari, M. and Mulu, B., 2013. Distribution of Trace Metals in Two Commercially Important Fish Species (Tilapia Zilli and Oreochromis Niloticus) Sediment and Water from Lake Gudbahri, Eastern Tigray of Northen Ethiopia, International Journal of scientific and Research Publications, vol. 3, pp. 2250-3153.

12. Mugidde, R., Gichuki, J., Rutagemwa, D., Ndawula, L., and Matovu, X. (2005). Status of water quality an its implication on the fishery production. In "The State of the Fisheries Resources of Lake Victoria and Their Management. Proceedings of the Regional Stakeholders' Conference" (L. V. F. O. Secretariat, ed.), pp. 106-12, Jinja, Uganda.

13. Spellman(2008), Handbook of water and wastewater treatment plant operations 2 nd edition

14. Tamiru, A., 2004. Assessment of Pollution Status and Groundwater vulnerability mapping of the Addis Ababa Water Supply Aquifers, Ethopia.

15. Tank, S. K. and Chippa, R.C., 2013. Analysis of Water Quality of Halena Block in Bharatpur Area, vol.3(3), International Journal of Scientific and Research Publications.

16. Weldemariam, M. M., 2013. Physico-chemical Analysis of Gudbahri River Water of Wukro, Eastern Tigrai, Ethiopia, International Journal of Scientific and Research Publications, vol.3(11).

17. Zhou, F., Guo, H.C., Liu, Y. and Jiang, Y.M., 2011. Chemometrics data analysis of marine water quality \& source identification in southern Hong Kong, Marine Pollution Bulletin 54 (6), pp. 745-756. 


\section{(Tables \& Figures)}

Fig 1, 2 and 3: Graph shows the analysed parameters of the lake water sample
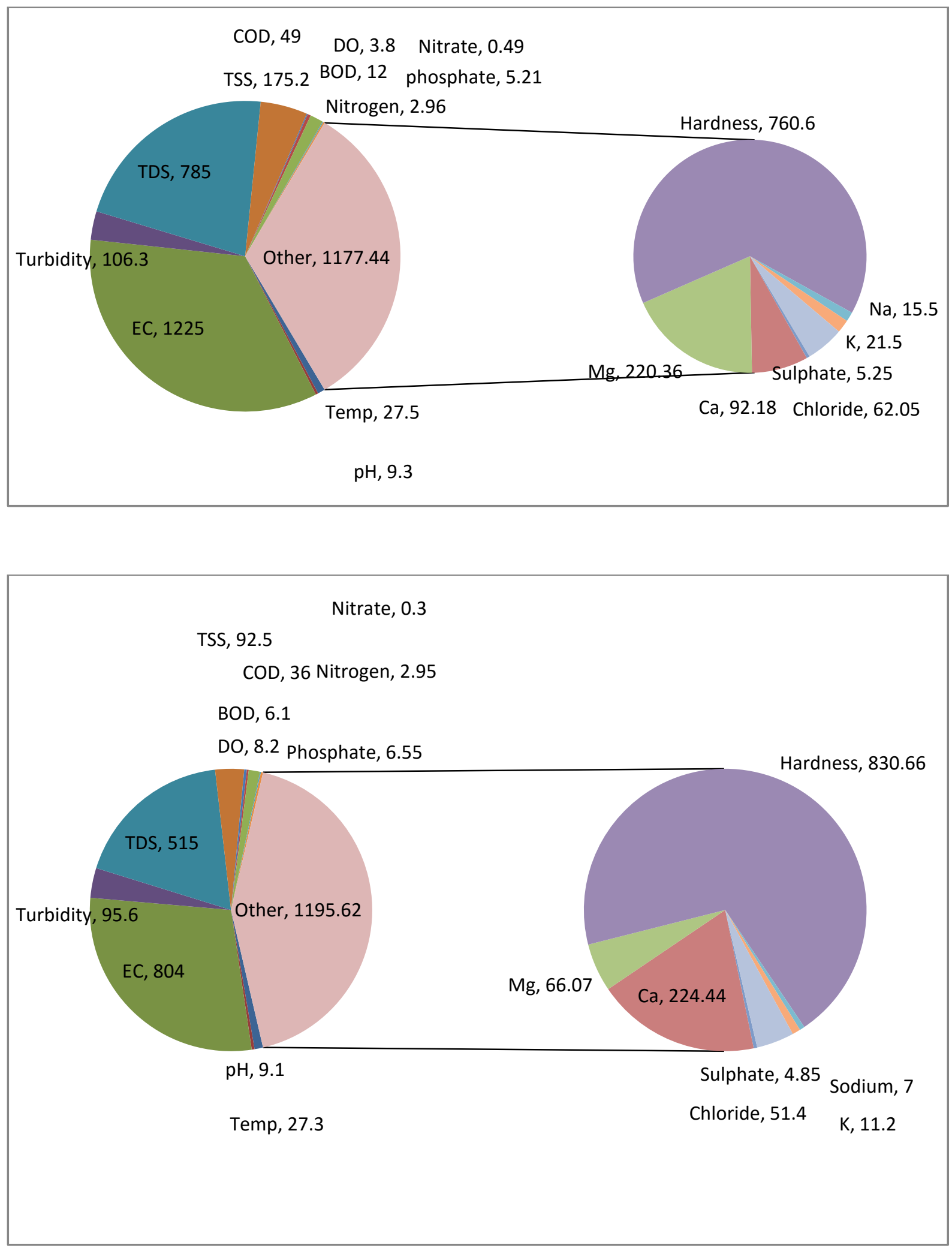


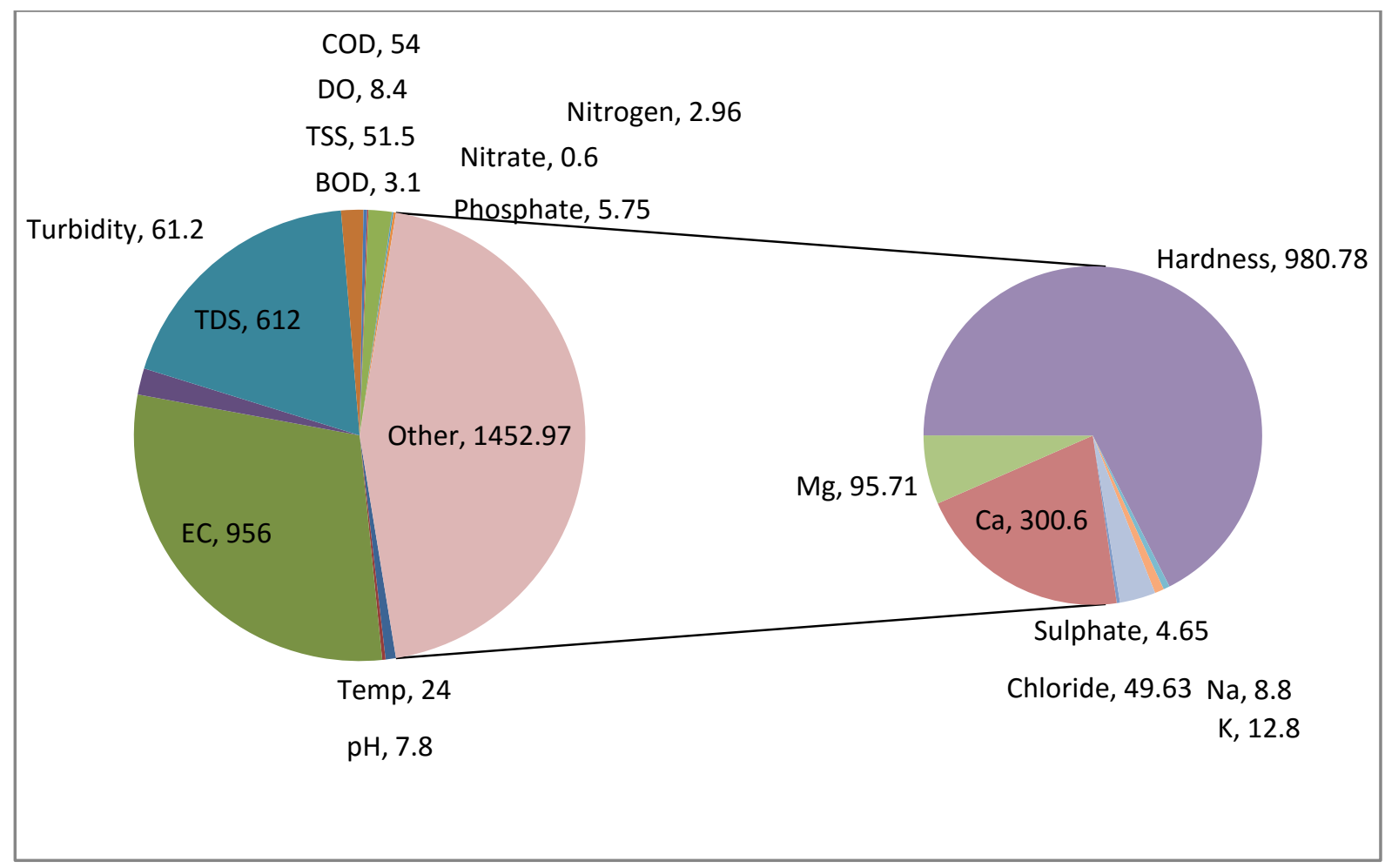

